\section{Avaliação do Intervalo de Tempo e Custo Médio para a Obtenção do Eutiroidismo na Doença de Graves Tratada com Drogas Antitiroidianas em Um Hospital Geral}

\section{RESUMO}

Os pacientes com doença de Graves tornam-se eutiróideos usualmente 6 a 12 semanas após iniciar os antitiroidianos. Aderência à medicação é vital para o sucesso da terapêutica. Com os objetivos de avaliar retrospectivamente o tempo necessário para a obtenção do eutiroidismo clínico e laboratorial e o custo da terapêutica clínica, analisamos 82 pacientes com doença de Graves atendidos entre fevereiro/96 e novembro/97 e acompanhados até julho/99. Destes, 49 (59,8\%) foram acompanhados até o eutiroidismo, ocorrido em 24,8 semanas. Apenas 11 alcançaram ○ eutiroidismo em 12 semanas. Dezesseis pacientes não aderentes despenderam tempo significantemente maior até o eutiroidismo que os aderentes ( 37,3 vs. 18,7 semanas). O abandono do tratamento ocorreu em $36,6 \%$ dos pacientes. O custo do tratamento em 24,8 semanas somou R\$248,72, contra $\mathrm{R} \$ 151,68$ em 12 semanas. Assim, nossos pacientes demoram mais tempo para alcançar o eutiroidismo que o usualmente descrito, implicando em um custo $64 \%$ maior. A falta de aderência é a justificativa mais importante para este atraso na compensação da tirotoxicose. (Arq Bras Endocrinol Metab 2001;45/6: 533-539)

Unitermos: Hipertireoidismo; Doença de Graves; Antitiroidianos; Gastos em saúde

\begin{abstract}
Most of the Graves' disease patients become euthyroid between 6 and 12 weeks after antithyroid drug use. Patients' compliance to the medication is essential for a successful therapy. The aims of the present study were to evaluate retrospectively the time spent to obtain clinical and hormonal euthyroidism, the costs of the clinical therapy in outpatients with Graves' disease and to compare them with the literature data. Eighty-two patients were selected between February/96 and November/97 and were followed-up until July/99. Forty-nine $(59.8 \%)$ returned regularly until reaching euthyroidism, which occurred at a mean time of 24.8 weeks. Only 11 patients were euthyroid in 12 weeks. Sixteen patients were considered no compliant and the time necessary to reach the euthyroidism was significantly higher than that of the compliant group ( 37.3 vs. 18.7 weeks). The rate of the patients who abandoned follow-up before reaching the euthyroidism was $36,6 \%$. The mean cost for 24.8 weeks treatment was $R \$ 248.72$ versus $R \$ 151.68$ for 12 weeks treatment, which is the period described in literature. In conclusion, our patients need more time to reach the euthyroidism than that usually described, which implies in $64 \%$ greater treatment cost. Poor compliance problems are the most important reason for this. (Arq Bras Endocrinol Metab 2001;45/6: 533-539)
\end{abstract}

Keywords: Hyperthyroidism; Grave's disease; Antithyroid agents; Health expenditures

\section{artigo original}

\author{
Roberta A. Nirschl \\ Fábio R. Kater \\ Sérgio O. Nascif \\ Alexandre E.F. Vieira \\ João C. Ramos-Dias \\ Maria-Helena Senger
}

Disciplina de Endocrinologia, Faculdade de Ciências Médicas de Sorocaba, PUC-SP, Sorocaba, SP.
Recebido em 14/08/00

Revisado em 09/04/01 e 13/07/01

Aceito em 14/08/01 
$\mathrm{A}$ DOEnÇA DE Graves É a FORMa mais comum de hipertiroidismo, e seus sintomas e sinais resultam da exposição dos tecidos à concentrações excessivas de hormônios tiroidianos (1-5).

A forma ideal de tratamento é difícil de ser estabelecida devido ao curso natural da doença ser longo, variável de um paciente para outro e também pela falta de marcadores precisos preditivos de remissão (6-8). As tionamidas (metimazol e propiltiouracil) são drogas antitiroidianas que têm sido usadas há mais de cinqüenta anos no tratamento de pacientes com hipertiroidismo e ainda representam a principal forma de terapia primária na doença de Graves (1,9-12).

Os antitiroidianos restituem o eutiroidismo de forma eficiente na fase inicial da terapêutica $(2,7,10,12,13)$. O tratamento é geralmente iniciado com doses elevadas dos agentes antitiroidianos que são progressivamente diminuídas conforme a melhora clínica e laboratorial do paciente $(2,12,13)$. Pode-se utilizar ainda uma terapêutica combinada de tionamidas e hormônios tiroidianos $(2,14)$. Vários fatores interferem no tempo de resposta à terapia clínica, incluindo a severidade da doença, o tamanho da tiróide e a posologia da medicação $(12,13)$. Porém, diversos estudos mostram que a maior parte dos pacientes se torna eutiróideo entre 6 e 12 semanas após o início da terapêutica $(2,6,12,13,15)$.

Como em qualquer tratamento clínico, a aderência do paciente ao tratamento proposto é vital para o seu sucesso. No período inicial do tratamento do hipertiroidismo isto é ainda mais importante, pois o mecanismo de concentração das drogas para atingir o nível terapêutico efetivo obriga que todas as tomadas diárias sejam efetuadas $(12,13)$. Além disto, como os níveis hormonais circulantes ainda estão elevados, esta é uma fase onde o paciente se encontra ainda susceptível às complicações e exacerbações do hipertiroidismo descompensado.

O objetivo do nosso estudo foi avaliar retrospectivamente o tempo necessário para a obtenção do eutiroidismo clínico e laboratorial e o custo da terapêutica clínica empregada na população com doença de Graves atendida nos ambulatórios de Endocrinologia do Conjunto Hospitalar de Sorocaba, Faculdade de Ciências Médicas de Sorocaba, PUC - SP.

\section{MATERIAL E MÉTODOS}

O presente estudo foi aprovado pelo Comitê de Ética em Pesquisa local. Foram analisados os dados obtidos em prontuários de pacientes portadores de hipertiroidismo devido a doença de Graves com confir- mação clínica e laboratorial. Tais pacientes fizeram sua primeira consulta no ambulatório de Endocrinologia entre os meses de fevereiro de 1996 a novembro de 1997 e seu seguimento foi avaliado até o mês de julho de 1999 (1,7 a 3,4 anos, média de 2,5 anos).

Para avaliar e mensurar os sintomas e sinais físicos do hipertiroidismo antes e durante o tratamento foi utilizada uma escala elaborada por Klein e cols. cuja soma máxima possível dos escores é de 40 (16) (tabela 1).

$\mathrm{O}$ diagnóstico laboratorial da doença de Graves baseou-se nas dosagens elevadas de T3 total (valor normal de 80 a $220 \mathrm{ng} / \mathrm{dl}$ ), e de T4 total (valor normal de 5 a $13 \mu \mathrm{g} / \mathrm{dl}$ ), além de níveis suprimidos de TSH (valor normal de 0,5 a $5,1 \mu \mathrm{UI} / \mathrm{ml}$ ) e títulos positivos de anticorpos antiperoxidase tiroidiana (valor normal: 1/100) e antitireoglobulina (valor normal: 1/100). As dosagens de T3 total, T4 total foram realizadas através de radioimunoensaio (DPC - Diagnostic Products Corporation, Los Angeles, CA) e as dos anticorpos antitiroidianos através do método Elisa. A determinação do TSH foi por método imunorradiométrico (DPC - Diagnostic Products Corporation, Los Angeles, CA; sensibilidade: $0,01 \mu \mathrm{UI} / \mathrm{ml}$ ). A dosagem do anticorpo anti-receptor de TSH não é disponível no serviço em que o estudo foi realizado.

Depois da consulta inicial, o primeiro retorno do paciente ocorreu após quatro semanas. A seguir, o intervalo médio entre as consultas foi de aproximadamente oito semanas, quando eram repetidas as dosagens de T3 total, T4 total e TSH. Através da aplicação da escala de sintomas e sinais e dos valores das dosagens hormonais foi determinado o tempo decorrido entre o diagnóstico do hipertiroidismo e a compensação eutiroidiana do paciente. Os pacientes foram considerados eutiróideos quando o escore clínico alcançou uma soma • a 7 e as dosagens de T3 e T4 se encontravam dentro dos valores normais.

Chamou-se de taxa de abandono, o percentual de pacientes que perderam o acompanhamento antes da obtenção do eutiroidismo.

Foram considerados como não aderentes os pacientes que tiveram ausência não justificada às consultas, uso irregular da medicação prescrita e/ou aqueles que não realizavam os exames laboratoriais periódicos solicitados.

O custo do tratamento até a obtenção do eutiroidismo foi calculado tomando-se o valor da consulta médica e exames laboratoriais pagos pelo Sistema Único de Saúde (SUS) na época do estudo. Segundo a tabela SUS vigente, o valor pago para cada consulta médica era de $\mathrm{R} \$ 2,55$. Cada um dos exames hormonais (T3 total, T4 total e TSH) custava $\mathrm{R} \$ 6,11$; para cada 
Tabela 1. Escala de sintomas e sinais do hipertiroidismo (16)

\section{Nervosismo:}

0. Ausente

1. Ansiedade somente sob "stress"

2. Ocasionalmente ansioso em repouso

3. Freqüentemente ansioso, dificuldade no trabalho e de concentração

4. Nervosismo a maior parte do tempo

\section{Sudorese:}

0. Somente com esforço físico

1. Em repouso, mas apenas em temperaturas moderadas

2. Em repouso, em clima ameno, principalmente nas mãos

3. Em repouso e em várias partes do corpo

4. Sudorese profusa, quase constantemente

\section{Tolerância ao calor:}

0. Normal

1. Eventualmente sente mais calor que os demais em um mesmo ambiente

2. Dificuldade significativa com o calor; usa ar condicionado constantemente no verão

3. Excessiva dificuldade com o calor, mesmo em temperaturas amenas

4. Extrema dificuldade com o calor; não se sente bem mesmo no inverno (não necessita de agasalhos ou cobertas)

Hiperatividade:

0. Normal

1. Atividade aumentada, com aumento da produtividade

2. Aumento da produtividade; menor tempo de sono

3. Faz algumas atividades inúteis

4. Episódios freqüentes de atividades inúteis; incapaz de permanecer sentado durante o exame

Tremor:

0. Ausente

1. Pouco perceptível

2. Tremor facilmente demonstrável ao exame físico

3. Tremor acentuado, mas hábil em moticidade fina

4. Mãos tremem excessivamente, dificuldade na motricidade fina

\section{Fraqueza:}

0. Força normal

1. Fraqueza subjetiva, porém com tolerância normal ao exercício

2. Menor tolerância ao exercício perto da atividade máxima

3. Dificuldade para subir escada ou levantar-se da cadeira

4. Fraqueza extrema; dificilmente carrega objetos ou sobe escadas

\section{Precórdio hiperdinâmico:}

0. Atividade e impulso apical normal

1. Taquicardia, $90 \mathrm{bpm}$, impulso apical normal

2. Taquicardia, $90 \mathrm{bpm}, \mathrm{com}$ aumento do impulso apical

3. Taquicardia, $110 \mathrm{bpm}$, impulso apical aumentado

4. Taquicardia, $110 \mathrm{bpm}$, impulso apical e pulso carotídeo aumentado, sopro sistólico

\section{Diarréia:}

0. 1 evacuação ao dia, fezes normais

1. 2 a 4 evacuações ao dia, fezes normais

2. 1 a 4 evacuações ao dia, fezes amolecidas

3. Mais de 4 evacuações ao dia, fezes normais

4. Mais de 4 evacuações ao dia, fezes amolecidas

Apetite:

0. Apetite normal, sem perda de peso

1. Apetite normal, com perda de peso

2. Apetite aumentado, sem perda de peso

3. Apetite aumentado, com perda de peso

4. Apetite diminuído, com perda de peso

\section{Avaliação das funções diárias} (grau de incapacitação):

\section{Ausente}

1. Incapacidade mínima (10\%)

2. Incapacidade leve (30\%)

3. Incapacidade moderada $(60 \%)$

4. Incapacidade severa (90\%) dosagem de anticorpos o valor era de R\$17,16. O cálculo do gasto com a medicação, a qual não era fornecida ao paciente, foi feito com base no preço do metimazol vigente em dezembro de 1999 nas principais farmácias da região de Sorocaba. Como o metimazol foi a medicação mais utilizada ( $92,7 \%$ dos pacientes), numa dose inicial de $45 \mathrm{mg}$ em $92 \%$ dos pacientes, consideramos o mesmo para fins de cálculo do custo diário médio com remédio, que foi de $\mathrm{R} \$ 0,90$.

Além da análise estatística descritiva dos dados, foi aplicado o teste de Mann-Whitney para comparação entre os pacientes aderentes e não aderentes em relação aos níveis iniciais de T3 e T4 totais e escore clínico, assim como para o tempo de obtenção do eutiroidismo clínico e laboratorial. O mesmo teste foi utilizado na comparação destes parâmetros entre os pacientes que usaram ou não $\beta$-bloqueador. $O$ coeficiente de correlação de Spearman foi utilizado para comparar os níveis iniciais de T3 e o tempo para obtenção do eutiroidismo. As diferenças foram consideradas estatisticamente significantes quando o valor de $\mathrm{p}$ foi menor que 0,05 . Os resultados estão descritos como média \pm erro padrão. 


\section{RESULTADOS}

Do total de 82 pacientes, 13 eram do sexo masculino $(15,9 \%)$ e 69 do sexo feminino $(84,1 \%)$. A idade do grupo variou de 15 a 77 anos (mediana de 33 anos). Pouco mais da metade dos pacientes $(54,5 \%)$ era procedente do município de Sorocaba.

O quadro clínico segundo a escala de sintomas e sinais físicos do hipertiroidismo e os níveis iniciais médios de T3 e T4 total, assim como os de TSH estão representados na tabela 2 .

Setenta e seis pacientes $(92,7 \%)$ foram tratados com metimazol, na dose inicial diária de 20 a $45 \mathrm{mg}$. Apenas 6 pacientes $(7,3 \%)$ fizeram uso de propiltiouracil na dose de $600 \mathrm{mg}$ ao dia. Um paciente apresentou náuseas e outro apresentou prurido como efeito colateral às drogas antitiroidianas, reações estas que não levaram à mudança ou suspensão da medicação. Vinte pacientes $(24,4 \%)$ fizeram uso de $\beta$-bloqueador adrenérgico (propranolol) no início do tratamento, na dose oral diária de $2 \mathrm{mg} / \mathrm{kg}$ de peso corporal. Um paciente não aderente foi a óbito devido a descompensação da doença (crise tireotóxica associada a cetoacidose diabética).

Quarenta e nove pacientes (59,8\%) mantiveram o acompanhamento até a obtenção do eutiroidismo clínico e laboratorial (figura 1). O escore médio, segundo a escala de sintomas e sinais físicos à época do eutiroidismo, foi de 2,0 $\pm 0,3$, variando de 0 a 6 . Os valores médios de T3 total e T4 total à época do eutiroidismo foram respectivamente $145,5 \pm 5,2 \mathrm{ng} / \mathrm{dl}$ e 9,5 $\pm 0,4 \mu \mathrm{g} / \mathrm{dl}$ e os de TSH $0,7 \pm 0,2 \mu \mathrm{UI} / \mathrm{ml}$ (tabela 2). O tempo médio decorrido até a compensação total do quadro foi de 24,8 semanas, variando de 4,6 semanas a 2,4 anos. Deste grupo de 49 pacientes apenas 11 $(22,4 \%)$ alcançaram o eutiroidismo clínico e laboratorial em até 12 semanas. Não houve correlação entre os níveis séricos iniciais de T3 e o tempo para obtenção do eutiroidismo $(p>0,05)$.

A taxa de abandono observada durante o período do estudo foi de $36,6 \%(n=30$ do total de 82 pacientes) (figura 1). Dos pacientes que abandonaram o seguimento antes da obtenção do eutiroidismo, $14 \mathrm{o}$ fizeram entre 1 e 3 meses após a consulta inicial.

Somente 2 pacientes não atingiram o eutiroidismo em nenhum momento durante o período de acompanhamento ( 1 e 2 anos), sendo ambos não aderentes à medicação (figura 1 ).

Dos 49 pacientes que alcançaram o eutiroidismo, $16(32,7 \%)$ foram considerados como não aderentes (figura 1). Os pacientes aderentes alcançaram o eutiroidismo em $18,7 \pm 2,2$ semanas e os não aderentes em 37,3 $\pm 7,3$ semanas. Houve diferença estatisticamente significante quanto ao tempo médio para a obtenção do eutiroidismo entre os pacientes aderentes e não aderentes $(\mathrm{p}=0,006)$, embora os valores iniciais de T3 e T4 totais e do escore clínico entre os dois grupos não tenham sido diferentes $(\mathrm{p}=0,51,0,66$ e 0,88 respectivamente).

Apenas 10 dos 49 pacientes que alcançaram o eutiroidismo fizeram uso de $\beta$-bloqueador e obtiveram o eutiroidismo num tempo significantemente maior $(\mathrm{p}=0,009)$ do que aqueles que não usaram propranolol (36,7 vs. 21,7 semanas, respectivamente). Os valores iniciais de T3 total entre os pacientes que fizeram uso ou não do $\beta$-bloqueador foram semelhantes $(\mathrm{p}=0,4)$.

Ao final do período de acompanhamento do estudo, 6 pacientes $(12,3 \%)$ estavam em remissão clínica e laboratorial (todos após uso de metimazol); $18(36,7 \%)$ foram encaminhados para tratamento definitivo com iodo radioativo (131I); $12(24,5 \%)$ continuavam em uso de antitiroidianos e $13(26,5 \%)$ abandonaram o seguimento após a obtenção do eutiroidismo (figura 1).

Considerando-se o tempo médio de 24,8 semanas para a obtenção do eutiroidismo clínico e laboratorial no grupo estudado $(\mathrm{n}=49)$, o custo médio do tratamento foi de R $\$ 248,72$ por paciente. Este custo foi reduzido para $\mathrm{R} \$ 151,68$ por paciente quando analisamos separadamente os pacientes que estavam compensados em até 12 semanas de tratamento $(n=11)$.

Tabela 2. Valores representados como média \pm desvio padrão do escore clínico (16) e dosagens hormonais iniciais $(n=82)$ e quando da obtenção do eutiroidismo $(n=49)$ em pacientes com doença de Graves.

\begin{tabular}{lcccc}
\hline & $\begin{array}{c}\text { Escore } \\
\text { clínico }\end{array}$ & $\begin{array}{c}\text { T3 } \\
(\mathbf{n g} / \mathrm{dl})\end{array}$ & $\begin{array}{c}\text { T4 } \\
\mu \mathrm{g} / \mathrm{dl})\end{array}$ & $\begin{array}{c}\text { TSH } \\
(\mu \mathrm{Ul} / \mathrm{ml})\end{array}$ \\
\hline INICIAL & $\begin{array}{c}12 \pm 0,3 \\
(7-22)\end{array}$ & $\begin{array}{c}512,2 \pm 23,3 \\
(140-850)\end{array}$ & $\begin{array}{c}26,4 \pm 0,8 \\
(14,3-44,1)\end{array}$ & $\begin{array}{c}0,1 \pm 0,01 \\
(0,01-0,24)\end{array}$ \\
\hline \multirow{2}{*}{ EUTIROIDISMO } & $2,0 \pm 0,3$ & $\begin{array}{c}145,5 \pm 5,2 \\
(87-201)\end{array}$ & $\begin{array}{c}9,5 \pm 0,4 \\
(6,2-12,9)\end{array}$ & $\begin{array}{c}0,7 \pm 0,2 \\
(0,1-4,4)\end{array}$ \\
\hline
\end{tabular}




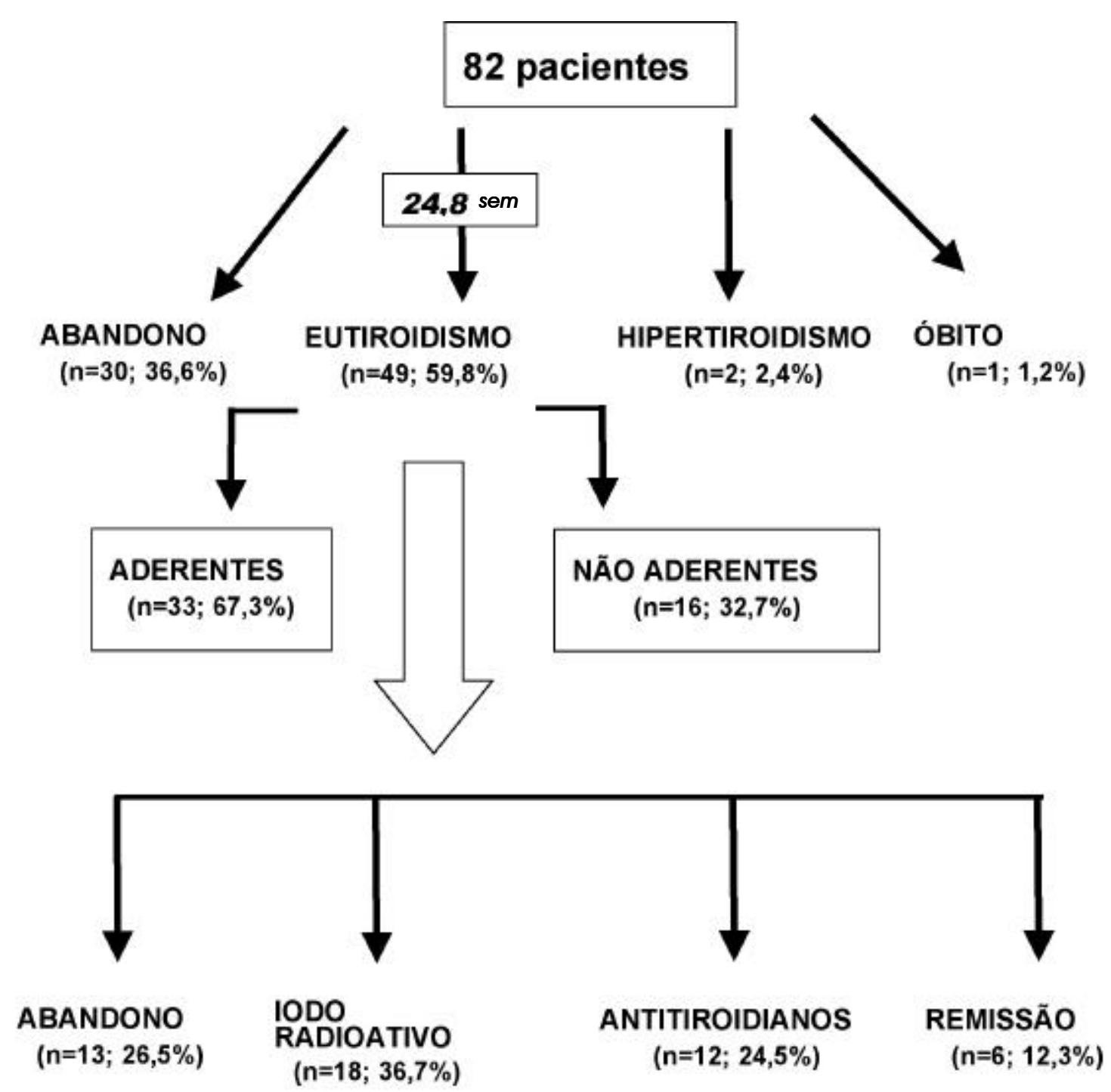

Figura 1. Evolução dos pacientes com doenças de Graves

\section{DISCUSSÃO}

Este estudo demonstrou que os pacientes com doença de Graves em acompanhamento no ambulatório de Endocrinologia do Conjunto Hospitalar de Sorocaba alcançam o eutiroidismo mais tardiamente $(24,8$ semanas) que o limite superior de tempo descrito em vários estudos da literatura (12 semanas) $(2,6,12,13,15)$. Isto implica em um tratamento cujo custo é aproximadamente $64 \%$ maior em nosso meio ( $\mathrm{R} \$ 248,72$ vs. $\mathrm{R} \$ 151,68)$.

Somente 11 dos 49 pacientes estavam em eutiroidismo na $12^{\mathrm{a}}$ semana após o início dos anti- tiroidianos, que é o tempo máximo apontado por vários estudos $(2,6,12,13,15)$. A falta de aderência dos pacientes foi considerada como a justificativa mais importante para este atraso na compensação do quadro de tirotoxicose. O que fortalece esta hipótese é a elevada taxa de abandono dos pacientes $(36,6 \%)$ antes da obtenção do eutiroidismo, com uma parcela significativa de pacientes $(\mathrm{n}=14)$ comparecendo a apenas uma ou duas consultas. Não temos conhecimento se estes pacientes continuaram seu tratamento em outro serviço. Pode-se supor que eles permanecem mais tempo susceptíveis às complicaçóes conseqüentes da exposição prolongada aos níveis circulantes elevados de 
hormônios tiroidianos, já que o atendimento terciário de nosso ambulatório é referência em uma região que abrange 48 municípios. Além disto, o único óbito ocorreu em um paciente que havia abandonado o tratamento. Portanto, ao maior custo do tratamento prolongado observado em nossos pacientes, soma-se o risco deste gasto ser ainda mais elevado devido às intercorrências às quais eles estariam sujeitos pela eventual permanência do quadro de tirotoxicose.

$\mathrm{O}$ uso de $\beta$-bloqueador não contribuiu para o alcance mais precoce do eutiroidismo. Aliás, neste estudo, notou-se que os pacientes que fizeram uso de $\beta$-bloqueador demoraram mais tempo para alcançar o eutiroidismo, apesar de não ter havido diferença estatística entre os níveis iniciais de T3 dos pacientes que usaram ou não o propranolol. Assim, a adição de mais um item na prescrição dos pacientes possivelmente contribua para a não aderência ao tratamento.

A má aderência pode ser conseqüência do menor nível de atenção e da labilidade emocional que são sintomas freqüentes nos pacientes com doença de Graves $(3,17)$. Outras causas que comprometem a aderência neste tipo de tratamento são a ingestão de um número elevado de comprimidos, a ocorrência de efeitos colaterais (embora este fator não tenha sido relevante no grupo estudado) e o fato da doença ser mais prevalente no sexo feminino, considerado mais indisciplinado e menos aderente à medicação $(18,19)$.

Em países desenvolvidos, pacientes com doenças tiroidianas que participam de grupos de apoio e associações apresentam melhora na qualidade de vida e no bem-estar emocional com redução da ansiedade e conseqüente aumento na aderência ao tratamento, o que pode reduzir os custos da terapêutica (20).

Em conclusão, a má aderência parece ser um fator preponderante no maior tempo necessário para a obtenção do eutiroidismo na doença de Graves em nosso meio. $\mathrm{O}$ fornecimento gratuito da medicação, a verificação do seu uso através da contagem de comprimidos, a disponibilidade de medicamentos de uso mais fácil, com posologias diferentes e/ou administração por vias diferentes, vida média sérica mais prolongada e concentração tiroidiana em dose eficaz por tempo mais prolongado e a implantação de grupos de apoio e educação ao paciente certamente contribuiriam para diminuir esta falta de aderência.

Controle clínico e metabólico mais eficientes, com menor índice de complicações representam significativa redução no custo total do tratamento, fator que deve ser considerado na indicação terapêutica e no manejo de portadores da doença de Graves.

\section{REFERÊNCIAS}

1. Larsen PR, Davies TF, Hay ID. The thyroid gland. In: Wilson JD, Foster DW, Kronenberg HM, Larsen PR, editors. Williams Textbook of Endocrinology. $9^{\text {th }}$ edition. Philadelphia: W.B.Saunders Company, 1998:389-515.

2. Romaldini $\mathrm{JH}$, Bromberg $\mathrm{N}$, Werner MC. Tratamento da doença de Graves com drogas antitiroidianas. Arq Bras Endocrinol Metab 1994;38:1-9.

3. Dabon-Almirante CLM, Surks MI. Clinical and laboratory diagnosis of thyrotoxicosis. Endocrinol Metab Clin North Am 1998;27:25-35.

4. Mclver B, Morris JC. The pathogenesis of Graves' disease. Endocrinol Metab Clin North Am 1998;27:73-89.

5. Graves PN, Davies TF. New insights into the thyroid-stimulating hormone receptor. The major antigen of Graves' disease. Endocrinol Metab Clin North Am 2000;29:267-86.

6. Leech NJ, Dayan CM. Controversies in the management of Graves' disease. Clin Endocrinol 1998;49:273-80.

7. Maugendre D, Gatel A, Campion L, Massart C, Guilhem I, Lorcy $\mathrm{Y}$, et al. Antithyroid drugs and Graves' disease prospective randomized assessment of long-term treatment. Clin Endocrinol 1999;50:127-32.

8. Orgiazzi J. Anti-TSH receptor antibodies in clinical practice. Endocrinol Metab Clin North Am 2000;29:339-55.

9. Lazarus JH. Hyperthyroidism. Lancet 1997;349-43.

10. Weetman AP. Graves' disease. N Engl J Med 2000;343:1236-48.

11. Wartofsky L, Glinoer D, Solomon B, Nagataki S, Lagasse R, Nagayama $Y$, et al. Differences and similarities in the diagnosis and treatment of Graves' disease in Europe, Japan, and the United States. Thyroid 1991;1:129-35.

12. Cooper DS. Antithyroid drugs for the treatment of hyperthyroidism caused by Graves' disease. Endocrinol Metab Clin North Am 1998;27:225-47.

13. Benker G, Vitti P, Kahaly G, Raue F, Tegler L, Hirche H, et al. Response to methimazole in Graves' disease. Clin Endocrinol 1995;43:257-63.

14. Hashizume K, Ichikawa K, Sakurai A, Suzuki S, Takeda T, Kobayashi $M$, et al. Administration of thyroxin in treated Graves' disease. N Engl J Med 1991;324:947-53.

15. Kallner G, Vitols S, Ljunggren J. Comparison of standardized initial doses of two antithyroid drugs in the treatment of Graves' disease. J Intern Med 1996;239:525-29.

16. Klein I, Trzepacz PT, Roberts M, Levey GS. Symptom rating scale for assessing hyperthyroidism. Arch Intern Med 1988; 148:387-90.

17. MacCrimmon DJ, Wallace JE, Goldberg WM, Streiner DL. Emotional disturbance and cognitive deficits in hyperthyroidism. Psychosom Med 1979;331-40.

18. Reilly JrPC, Reilly MC. The cost of outpatient physician's services at a Veterans Administration Hospital. Ann Intern Med 
$1980 ; 93: 128-32$

19. Ward LS, Castelo Filho A, Menabó E, Ribeiro SRR, Lima MC, Maciel RMB. Estudo da relação custo/efetividade no tratamento da doença de Basedow-Graves. Rev Assoc Med Bras 1986;32:147-54.

20. Wood LC. Support groups for patients with Graves' disease and other thyroid conditions. Endocrinol Metab Clin North Am 1998;27:101-7.

Endereço para correspondência:
Maria Helena Senge

Av. São Paulo 2918

18.013-004 Sorocaba, SP

fax: (15) 227-2565

e.mail: mhsenger@dglnet.com.br 